\title{
I. INVITED LECTURES AT PLENARY SESSIONS
}

\section{ON BIORHEOLOGY}

\author{
A. L. Copley* \\ 50 Central Park West, New York, N.Y. 10023
}

Hemorrhage and Thrombosis Research Laboratories, Department of Pharmacology, New York Medical College, New York, N.Y. 10029 and Bioengineering Group, Newark College of Engineering, Newark, N.J. 07012.

Descriptions of biorheological phenomena go back into antiquity, but studies of flow properties of living matter began probably with the discovery of the circulation in blood capillary vessels by Malpighi in 1686 and the streaming in plant cells by Corti in 1774. Biorheology comprizes the study of the deformation and flow of living organisms and inanimate biological systems or of materials directly derived from living organisms. The term biorheology was introduced in 1948 (A. L. Copley, Proc. Internat. Congress on Rheology, Scheveningen, Holland, 1948, North-Holland Publ. Co., Amsterdam and Interscience Publishers, New York, 1949, Vol. 1, p. 47). Ever since, biorheology offered a framework to connect the sciences of biology with rheology. This frame, which proved to be secure, permits the application of a number of rheological treatments to biological systems. The different fields of biorheology, including hemorheology, have grown rapidly and to such an extent that any abridged survey will remain inadequate. Jean-LéonardMarie Poiseuille first reported in 1835 his in vivo studies "Recherches sur les causes du mouvement du sang dans les vaisseaux capillaires", which led to the application of rheological treatments to the flow of blood. The discovery of the laws of flow was based on experiments which Poiseuille was stimulated to make from his observations in living blood capillaries, resulting in his studies "Recherches expérimentales sur le mouvement des liquides dans les tubes de tres petits diamètres". These reports, published from 1840 to 1842 , were accepted throughout Europe. On the basis of Poiseuille's findings, Maxwell, Jacobson Mathieu and others deduced from the fundamental equation of Newton the well known formula for viscosity, which was later named after Poiseuille. It will be demonstrated that there are other reciprocal stimuli from biology and rheology, which promise to continue to be fruitful. Numerous biological phenomena and processes await a rheological approach for the characterization of the flow properties involved and for quantitative studies. It will be shown that biorheology, in spite of its brief history as an organized science, is of growing importance in the biological and medical sciences. New knowledge gained in biorheology, as applied to the practice of medicine and surgery, will serve the well-being of the human species.

\section{SOME MICRORHEOLOGICAL ASPECTS OF PLATELET THROMBOSIS}

\author{
H. L. Goldsmithi \\ University Medical Clinic, Montreal General Hospital, Montreal 109, Canada
}

OBSERVATIONS in vivo and in vitro have demonstrated the presence of platelet thrombi on vessel walls downstream from bifurcations, obstructions and sharp bends. Since flow at these sites is disturbed and separation of streamlines from the walls with vortex formation can occur, fluid mechanical factors have been cited as a cause of thrombus formation. To elucidate the effects of flow patterns in blood on the behavior and interaction of individual corpuscles, studies were undertaken to determine (1) how, in view of the known migration of blood cells away from vessel walls, do platelets come into the proximity of the endothelium and (2) whether conditions at sites of flow separation are conducive to thrombus formation.

(1) Particle paths near vessel walls. These were studied using $2 \mu$ latex spheres as models of platelets in transparent plasma suspensions of reconstituted biconcave ghosts at volume concentrations of 4-50 per cent flowing through glass tubes of $100 \mu$ diameter. Cell interactions led to continuous, often large displacements in sphere paths normal to the direction of flow. This resulted in numerous collisions with the wall suggesting a mechanism by which platelets can interact with the endothelial cell surface.

(2) Flow at an obstruction. Dilute suspensions of red cells and latex spheres were observed flowing past model spherical protuberances on the walls of $1 \mathrm{~mm}$ glass tubes at Reynolds numbers from 20 to 50 . A vortex formed downstream through which particles orbited in spiral paths taking a relatively long time to rejoin the mainstream. Shear rates at the tip of the spherical obstacles were several times greater than wall shear

BIo. 9/3-B 
rates far from the obstacle. In contrast, near the wall in the reverse flow region, shear rates and velocities were very much lower and aggregates of latex spheres adhered to the glass. Conditions in the vortex thus may make it possible for platelets, once activated to form aggregates and adhere to the vessel wall.

(Supported by Grant MT 1835 of the Medical Research Council of Canada.)

\title{
BIORHEOLOGY, INFORMATION THEORY AND CYBERNETICS
}

\author{
George Bugliarelio* \\ College of Engineering, University of Illinois at Chicago Circle, Chicago, Illinois
}

BIORHEOLOGY has been concerned traditionally with a certain set of constitutive properties of biological fluids, which are used to analyze or predict momentum, mass and heat exchange processes in the fluids of living systems. Such processes, however, have not only the function to energize the living systems but also to convey information-through pressure pulses, the conveyances of heat and above all, the conveyance of hormonal (chemical) signals.

An understanding of the characteristics of these information conveyance mechanisms is unfortunately extremely limited at this moment. Biorheology can contribute to such an understanding through studies of the most appropriate rheological characterization of biological fluids in terms of the information conveyance processes, and through studies of the role played in such processes by different rheological characteristics.

In turn, focus on the information conveyance properties of biological flows can be the point of departure for the introduction of cybernetics considerations. In the first place, can cybernetics contribute to an understanding of constitutive characteristics of biological fluids? Secondly, what is the role that the rheological characteristics of biological fluids play in the cybernetics processes of living systems?

Because of large gaps in our knowledge of rheological properties and information control processes in living systems, none of these questions can be answered today to any significant depth. However, what can be done is to map a systematic program of investigations that will hopefully provide such answers.

\section{A RHEOLOGICAL STUDY OF THE TRANSITION REGION FROM CONTINUOUS DEFORMATION TO BREAKUP AND ITS BIOLOGICAL SIGNIFICANCE}

\author{
K. WEISSENBERG $\dagger$ \\ Ridderlaan 111, Den Haag, Holland
}

THE FRACTURE of a bone or the rupture of a blood vessel can constitute a serious condition which may lead to a grave illness, or even to paralysis and sudden death. Preceding the actual break there is a transition region from continuous deformation, and a rheological study of this region in industrial processes reveals the existence of materials of all kinds of consistencies and conditions for which the said region displays danger signals in the form of the rheological phenomena of fatigue, dilatancy, work hardening and embrittlement. The said phenomena possess various unexpected, and apparently paradoxical phenomena some of which will be demonstrated. If and when a danger signal is observed there is sometimes the possibility to take action which prevents the breakage, relaxes the fatigue and produces negative dilatancy, work-softening and fluidification.

For biological materials there are, unfortunately, no data available as yet, but one may expect to encounter phenomena similar to those mentioned above for many biological materials, particularly under some pathological conditions as e.g. for calcified arteries. The observation of a danger signal and the possibility of taking preventive action are here, of course, of special interest.

\section{MODELLING THE MECHANICAL BEHAVIOR OF RED BLOOD CELLS}

\author{
RICHARD SKALAK $\dagger$
}

Department of Civil Engineering and Engineering Mechanics, Columbia University, New York, N.Y. 10027

THE HUMAN red blood cell is known to consist of a thin, flexible membrane filled with a concentrated solution of hemoglobin which has been shown to behave as a Newtonian fluid. The plasma in which red blood cells 
are suspended is also Newtonian. The properties of the red blood cell membrane are less well known and there are estimates of elastic moduli reported in the literature differing by as much as a factor of $10^{3}$. The lowest elastic modulus appears in uniaxial tension tests in which one of the principal stresses in the membrane is zero. The largest moduli result from tests in which the tension in the membrane is isotropic, such as in sphering of red blood cells, near hemolysis. It is shown that these disparate moduli can be incorporated into a single description of the red blood cell membrane by use of the notion of the strain energy function. The derivatives of the strain energy function with respect to the Green strain components yield the stress components. The expressions are valid for large strains such as occur in red cell membranes in sphering and sieving experiments. The main features of the red cell membrane that emerge are that it is much stiffer with respect to changes of areas than for strains in which the area is constant. The red blood cell membrane has also been shown to be viscoelastic to some extent and such behavior is incorporated by a modification of the stress-strain relations derived by use of the strain energy function. Examples of computations made with the proposed modelling of the red blood cell are shown for sphering, sieving and pipette experiments and the flow of red blood cells in narrow capillaries.

\title{
RHEOLOGICAL PROPERTIES OF LIVING SOFT TISSUES \\ YuAn-Cheng B. Fung $\dagger$ \\ University of California, San Diego, La Jolla, California 92037
}

To UNDERSTAND the physiological function of vital organs we must know the mechanical properties of the tissues. Experimental determination of the mechanical properties of living tissues has many difficulties, such as the small size, large deformation, active contraction, damage due to dissection, inaccessibility of "natural" state and the necessity of keeping the specimens alive. In this paper, results obtained in our laboratory on the rheological properties of soft tissues are summarized. This includes connective tissues, blood vessels, heart muscles, ureter and others. From these results a mathematical description of the behavior of normal tissues is offered to serve as a starting point for the analysis of the function of the organs.

\section{THE DYNAMIC RIGIDITY OF FIBRIN GELS}

EIICHI FuKada $\dagger$ and MaKoto KaIBARA

The Institute of Physical and Chemical Research, Wako, Saitama, 351, Japan

THE DYNAMIC rigidity modulus and loss modulus were determined with a viscoelastorecorder for the fibrinogen solutions clotted by adding thrombin. The dynamic rigidity of the fibrin gel depends upon the amplitude and frequency of oscillation applied during coagulation. The logarithm of rigidity decreases in proportion to the square root of the dynamic shear rate, i.e. the frequency times amplitude. The mechanical disturbance during clotting prevents the sufficient formation of the fibrin network. The rigidity of the gel also increases in proportion to the square of the concentration of fibrinogen.

The clotting curves are represented by the superposition of two kinetic processes: one is the crosslinking reaction between fibrin polymers, and the other is the stretching of molecular chains between crosslinkages. After the completion of gelation, if the amplitude of oscillation is decreased, the rigidity modulus decreases until it reaches to the value of the unelongated network. With such small amplitude the rigidity increases with the rise of temperature around room temperature, suggesting the rubber-like elasticity in the unstretched fibrin gels.

\section{THE PATHOLOGICAL RED CELL AGGREGATION (BLOOD SLUDGE): MICRORHEOLOGY AND PROTEIN CHEMISTRY}

\author{
H. SCHMID-SCHÖNBEIN† \\ Department of Physiology, University of Munich, German Federal Republic
}

THE OBSERVATION of aggregated red blood cells in the living microcirculation in man (blood sludge) is one of the most common hemorheological abnormalities in clinical medicine. Although a classical subject of hemorheology, it has never been quantified adequately in vivo nor in vitro. Attempts to correlate the pathological red cell aggregation to the erythrocyte surface charge, to the suspension stability and to the apparent 
viscosity of blood failed to clarify the hemodynamic significance of the phenomenon. It is in fact not established beyond doubt whether the microcirculatory retardation is a cause or consequence of the intravascular aggregation.

The newer concepts and methods of blood microrheology have now been employed to compare quantitatively the flow behavior of normal human blood and that of myeloma patients exhibiting pathologically intensified red cell aggregation. Rotational viscometry, the photometric recording of cell aggregation and cell orientation in viscometric flow were combined with direct microscopic observation of the blood subjected to viscometric flow in a transparent cone plate chamber. In this chamber, the cone is in counterrotation to the plate: although subjected to quantified shear stresses in viscometric flow, the cells remain stationary to the observer and can be studied at magnifications up to $1000 \times$ in dark field, bright field and phase contrast illumination. In the same instrument, the shear stresses of hydrodynamic desegregation, the rate of aggregate formation in stasis and in slow flow $\left(<0.5 \mathrm{dyn} / \mathrm{cm}^{2}\right)$ are quantified photometrically.

Both in normal and in pathological blood, red cells are aggregated into rouleaux at shear stresses below $1 \mathrm{dyn} / \mathrm{cm}^{2}$, with decreasing shear, these form secondary structures, and give rise to a steep increase in apparent viscosity in prestatic flow. In normal human blood rouleaux networks form due to end-to-side attachment. In pathological blood, coarse clumps of short rouleaux are formed due to side-to-side attachment. These latter structures are not only much more resistant against shear (hydrodynamic desegregation at 5-20 dyn $/ \mathrm{cm}^{2}$ ), but they rapidly increase in size at shear stresses below $1 \mathrm{dyn} / \mathrm{cm}^{2}$ in spite of constant shearing. After hydrodynamic desegregation, both the normal and the pathological aggregates undergo a fluid droplike transition, which is responsible for the unusually low apparent viscosity of blood, especially at high hematocrits and in narrow tubes.

The pathological aggregation may be caused by either increased plasma fibrinogen content, or more commonly, by the presence of non-clottable serum proteins. The aggregating serum proteins were isolated by gel and glass-chromatography, free flow electrophoresis and isoelectric focussing, tested for activity in the counter-rotating cone plate chamber and then identified immunologically. In the present group of myeloma patients, $a_{2}$-macroglobulins and IgM were the only proteins exhibiting aggregating effects. Distinct microrheological differences between these two proteins were found: red cells in purified $\alpha_{2}$-macroglobulin solutions formed extremely shear resistant, tight aggregates, while red cells in IgM showed strong aggregation only in stasis.

Due to the fluid drop-like flow behavior of dispersed red cells, increases in plasma viscosity favor hydrodynamic desegregation. All other factors remaining constant, the shear stresses necessary for hydrodynamic desegregation rise with falling hematocrit value. This surprising finding stands in contrast to the effect of reduced hematocrit on apparent blood viscosity in prestatic flow, which, of course, is markedly reduced with lowered hematocrit.

The present results substantiate the concept that the low shear stresses acting in the post-capillary venules predispose these vessels as the site of intravascular aggregate formation. The paradox effect of the hematocrit on shear resistance of aggregates and on the apparent blood viscosity may explain why in many patients exhibiting the combination of anemia and pathological red cell aggregation intravascular sludging is observed without overt clinical consequences thereof.

Supported by the Deutsche Forschungsgemeinschaft. 